\title{
Tissue Reconstruction Based on Deformation of Dual Simplex Meshes
}

\author{
David Svoboda and Pavel Matula \\ Laboratory of Optical Microscopy \\ Faculty of Informatics, Masaryk University \\ Botanická 68a, 60200 Brno, Czech Republic \\ \{xsvobod2, pam\}@fi.muni.cz
}

\begin{abstract}
A new semiautomatic method for tissue reconstruction based on deformation of a dual simplex mesh was developed. The method is suitable for specifically-shaped objects. The method consists of three steps: the first step includes searching for object markers, i. e. the approximate centre of each object is localized. The searching procedure is based on careful analysis of object boundaries and on the assumption that the analyzed objects are sphere-like shaped. The first contribution of the method is the ability to find the markers without choosing the particular objects by hand.

In the next step the surface of each object is reconstructed. The procedure is based on the method for spherical object reconstruction presented in [3. The method was partially changed and was adapted to be more suitable for our purposes. The problem of getting stuck in local minima was solved. In addition, the deformation process was sped up.

The final step concerns quality evaluation: both of the first two steps are nearly automatic, therefore the quality of their results should be measured.
\end{abstract}

Keywords: Deformable models, dual simplex mesh, quality evaluation, reconstruction.

\section{Introduction}

Various pieces of experience in analyzing biomedical images show that tissue cells are quite heterogenous. Every cell is different and it is difficult to find a universal image analysis approach. The main characteristics such as similarity in shape, continuity of surface, volume, etc. are the only guide to choose which objects in the image could be supposed to be a cell and which couldn't.

Many techniques for the reconstruction of particular cells have been developed. Some of them have used thresholding [74], some others deformable models. It seems better to choose the deformable models as the way of object reconstruction because these techniques are capable of working with a priori knowledge about the shape of an object. They can handle missing or noisy data.

For the first time, the deformation of curves in 2D was introduced by Kass et al. 6], who proposed active contours model. The idea was based on the successive 
deformation of the curve, defined by differential equations, until the stable state was reached. This model is very suitable for reconstruction of circular objects. Therefore Bamford \& Lovell [1] applied it to biomedical data, especially cells. They simplified this method and improved it by solving problem of touching or overlapping cells. Unfortunately the extension of these methods to 3D space is nontrivial. Another approach has been chosen by Delingette. He proposed simplex meshes [2] and suggested to use them as a data model suitable for deformation. In his work the objects of general shape were reconstructed. Later Matula \& Svoboda [3] used this idea and applied it to biomedical images. They have developed the idea and made it more suitable for the purpose of biomedical images.

Unfortunately not many techniques for tissue reconstruction have been developed yet. Nowadays the reconstruction of biomedical objects based on mathematical morphology is very often used [1513. Also another technique based on deformable models was proposed. Sethian introduced level sets [8] based on moving general surface defined by partial differential equations. The idea was used by Solorazno et al., who presented this approach in tissue segmentation [5].

In this paper a new semiautomatic three-step method for tissue reconstruction is described. It is based on the deformation of a dual simplex mesh. This simplex mesh has the same meaning as the star-shaped simplex mesh showed in 3. The attribute "dual" origins in the dual active contour method presented by Gunn 10. Due to the duality of the mesh, setting the initial state of the deformed surface is simpler than in previous methods. Therefore, it seems to be convenient for tissue segmentation.

Dual simplex mesh is defined in Section 2.1] Next the deformation principle and searching for energy minima of the mesh is presented. The application to real biomedical data is demonstrated. The results are discussed in Section 3

\section{The Method}

\subsection{Definition of Dual Simplex Mesh}

Definition 1. Let $M$ be an arbitrary star-shaped simplex mesh [3] with the centre $Q$. Let $\mathcal{H}\left(Q, q_{i}\right)$ be homotheties with the centre $Q$ and ratios $q_{i}, q_{i} \in<1, \infty>$, $\forall i \in\{1,2, \ldots, n\}$, where $n$ is the number of vertices in $M$. Let $M^{\prime}$ be another star-shaped simplex mesh such that $\forall P_{i}{ }^{\prime} \in M^{\prime} ; P_{i}{ }^{\prime}=\mathcal{H}\left(Q, q_{i}\right)\left(P_{i}\right) ; P_{i} \in M ; i \in$ $\{1, \ldots, n\}$ and each $P_{i}^{\prime}$ has the corresponding neighbours $P_{i_{N 1}}^{\prime}, P_{i_{N 2}}^{\prime}$ and $P_{i_{N 3}}^{\prime}$. The union $D=M \cup M^{\prime}$ is called dual simplex mesh.

Note 1. All the vertices in $D$ have the same properties as the vertices in the general simplex mesh.

Note 2. Let us introduce the symbols used in the following text: The symbol $D$ is used for any dual simplex mesh. $M$ is called inner mesh and its mapping $M^{\prime}$ is called outer mesh. The vertex $P_{i} \in M$ and its mapping $P_{i}{ }^{\prime} \in M^{\prime}$ are called counterparts. The abscissa $l_{i}$ connecting them is called connection line of vetrices $P_{i}$ and $P_{i}{ }^{\prime}$. 


\subsection{Deformation of Dual Simplex Mesh}

Suppose the vertices in the mesh can be moved using some of law of motion and suitable forces. Therefore the arbitrary movement is not permitted. In the following text the law of motion and principle of deformation of the dual simplex mesh are presented.

Let $P_{i} \in M$ be any vertex and $P_{i}{ }^{\prime} \in M^{\prime}$ its counterpart. The motion of vertex $P_{i}$ is allowed along the ray $\overrightarrow{P_{i} P_{i}^{\prime}}$ towards $P_{i}^{\prime}$ only. Vice versa, the motion of vertex $P_{i}^{\prime}$ is allowed along the ray $\overrightarrow{P_{i}{ }^{\prime} P_{i}}$ towards $P_{i}$ only. Now let us define the two energies for each vertex in a dual simplex mesh (shortly mesh):

Internal Energy. As mentioned above, for any vertex $P_{i}$ it is possible to define many of its geometric properties. The simplex angle $\varphi_{i}$ is the most important one. According to the values of $\varphi_{i}$ the shape of the mesh in the local neighbourhood of vertex $P_{i}$ and consequently the relative position of vertex $P_{i}$ to its neighbours can be defined. Namely the local curvature and continuity of n-th (for any natural number $n$ ) derivative of the surface represented by the mesh are known. Vice versa, if the local curvature or derivative in vertex $P_{i}$ is known (e. g. some specific smoothness of object surface is required) the desired simplex angle $\tilde{\varphi}_{i}$ is known and the position of the vertex $P_{i}$ can be set to fulfil the appropriate requirements. The desired simplex angle is called reference simplex angle. Then, every time the surface is arbitrarily deformed, the shape constraint pushes the surface to the state required by $\tilde{\varphi}_{i}$.

There are several constraints applied to the surface smoothness [3]. In the following enumeration the two most important of them are presented:

- shape constraint. $\tilde{\varphi}_{i}$ is usually set to constant value $\varphi_{i}^{0}$ (zero index says it is the value of the simplex angle in the initial state of the mesh - before any deformation).

- $C^{2}$ constraint. $\tilde{\varphi}_{i}$ is set to the average value of the simplex angles at neighbouring vertices.

Now, let $\tilde{\varphi}_{i}$ be the reference simplex angle bearing a constraint for the vertex $V_{i}$ in mesh $D$ and let $\varphi_{i}$ be the current simplex angle. The internal energy $E_{\text {int }}\left(V_{i}\right)$ in vertex $V_{i}$ is defined as:

$$
E_{\text {int }}\left(V_{i}\right)=\left\{\begin{array}{l}
\left(\frac{\varphi_{i}-\tilde{\varphi}_{i}}{2 \pi}\right), V_{i} \in M \\
\left(\frac{\tilde{\varphi_{i}}-\varphi_{i}}{2 \pi}\right), V_{i} \in M^{\prime}
\end{array}\right.
$$

and expresses the tension between the current position of vertex $V_{i}$ with its simplex angle $\varphi_{i}$ and desired position of this vertex presented by the reference simplex angle $\tilde{\varphi}_{i}$.

External Energy. Let $l_{i} \in D$ be any connection line between its vertices $P_{i}, P_{i}{ }^{\prime}$. Furthermore let image data be represented by the two image buffers: 


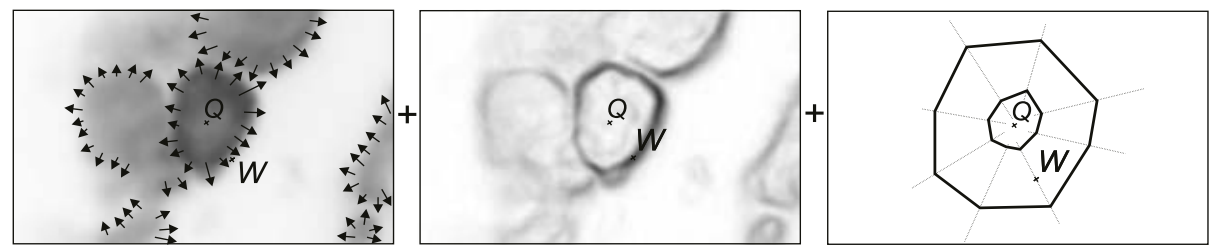

Fig. 1. Composition of image gradient direction (left), gradient image (center) and a dual simplex mesh (right). For any vertex $W \in \mathbb{R}^{3}$ the intensity of gradient value and gradient direction can easily be acquired.

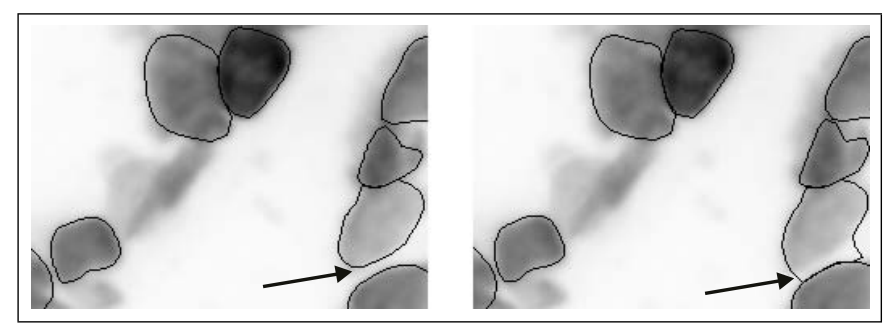

Fig. 2. (left) Deformation with image gradient direction is correct. (right) Incorrect result - without using gradient direction.

- I . . simply preprocessed (smoothing, median) data

- $|\nabla I| \ldots$ gradient image evaluated from $I$

Let us put all these data structures together (see Fig. 11), so that each particular voxel $W \in l_{i}$ has its own $I(W)$ and $\nabla I(W)$ values as well as its local neighbourhood. The external energy $E_{\text {ext }}(W)$ in voxel $W$, which belongs to connection line $l_{i}$ with direction vector $\boldsymbol{v}$, is defined by:

$$
E_{\text {ext }}(W)=1.0-\delta_{W, \boldsymbol{v}}\left(\frac{|\nabla I(W)|}{\max (|\nabla I|)}\right),
$$

where

$$
\delta_{W, \boldsymbol{v}}= \begin{cases}1, & \nabla I(W) \cdot \boldsymbol{v}>0 \\ 0, & \text { otherwise }\end{cases}
$$

and $\max (|\nabla I|)$ is maximal possible value of $|\nabla I|$. Using the division, the value of external energy is normalized.

Hence the external energy in voxel $W$ represents the importance of gradient and its direction in image data lying at the position of voxel $W$. The lower the value of the external energy the more stable the position of any vertex occupying the place of voxel $W$ is. The reason of using image gradient direction is explained in Figure 2 A similar problem has been solved in [1.

It can be seen, that after computing the external energy in each voxel of connection line $l_{i}, \forall i \in\{1, \ldots, n\}$ both buffers $I$ and $|\nabla I|$ can be removed. This step has two advantages: 
1. There is no need of computing the external energy later during the deformation. Everything has already been precomputed.

2. The image buffers are about $1300 \times 1030 \times 40$ voxels stored in main memory. Hence the permanent allocation of this huge memory block is undesirable.

Now the two different energies are defined. Internal energy represents the connectivity of mesh. External energy expresses the important voxels in image space.

Law of motion. The same law of motion as in general simplex meshes [2] and star-shaped simplex meshes [3] was used. Vertices of a mesh are considered as physical mass submitted to a Newtonian law of motion:

$$
m \frac{d^{2} P_{i}}{d t^{2}}=-\gamma \frac{d P_{i}}{d t}+\mathbf{F}\left(P_{i}\right)
$$

where $m$ is the vertex mass and $\gamma$ is the damping factor [2]. F is the force applied to each vertex and is defined in the following text. The evolution of the mesh in time under this law of motion can be discretized. It is done by using central finite differences:

$$
P_{i}^{t+1}=P_{i}^{t}+(1-\gamma)\left(P_{i}^{t}-P_{i}^{t-1}\right)+\mathbf{F}\left(P_{i}\right)
$$

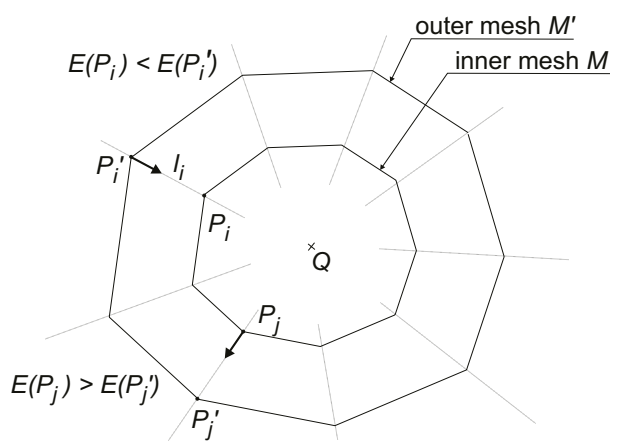

Fig. 3. Force definition: Each vertex moves towards its counterpart only. The movement is enabled/disabled according to the values of potential energies of particular vertices.

The principle is explained in Figure 3. For each connection line $l_{i}$ between the corresponding vertices $P_{i}$ and $P_{i}^{\prime}$ in the mesh the four energies could be computed: $E_{\text {int }}\left(P_{i}\right), E_{\text {ext }}\left(P_{i}\right), E_{\text {int }}\left(P_{i}^{\prime}\right), E_{\text {ext }}\left(P_{i}^{\prime}\right)$. They are used in this way:

$$
\begin{aligned}
E\left(P_{i}\right) & =\alpha E_{\text {int }}\left(P_{i}\right)+(1-\alpha) E_{\text {ext }}\left(P_{i}\right) \\
E\left(P_{i}^{\prime}\right) & =\alpha E_{\text {int }}\left(P_{i}^{\prime}\right)+(1-\alpha) E_{\text {ext }}\left(P_{i}^{\prime}\right)
\end{aligned}
$$

where $\alpha \in<0 ; 1>$ is a parameter affecting the smoothness of reconstructed object. The force $\mathbf{F}$ is defined as a shrinkage of the connection lines $l_{i}$ : Let $l_{i}$ be any connection line with its vertices $P_{i}$ and $P_{i}{ }^{\prime}$. One of them has higher energy and therefore is less stable. This vertex is shifted towards its counter part. If both of them have the same energy level, the outer one is chosen for motion. 


\subsection{The Algorithm}

Inputs. When proposing this method, the aim was to eliminate the necessity of choosing many parameters and particular objects by hand. Therefore the algorithm requires the following input only: image data, minimal and maximal diameter $\left(d_{\text {in }}\right.$ and $d_{\text {out }}$, respectively) of studied objects, required smoothness $\alpha$ and damping factor $\gamma$.

Outputs. The set of appropriately deformed dual simplex meshes (see Fig. 60) is returned. The contour lines (see Fig. 15 (right)) drawn back onto source data to view the exact shape of reconstructed surface is optional. In this way, the results can easily be observed.

The presented method is semiautomatic and consists of three steps:

Searching for Markers. The aim of this step is to find object markers (see Fig. 5 (left)). In this case the tissue cells are the objects. One marker belongs to one objects and is its approximate center of gravity. There should not be two or more markers matching one object. Nearly one-to-one correspondence between markers and cells is expected. The procedure is defined in Figure 4

1. Read the image data and parameters from an input and create convolution kernel $K$ in the shape of sphere with radius $d_{i n}$.

2. buf $\leftarrow|\nabla I| \otimes K$

3. buf $\leftarrow I \backslash$ buf

4. buf $\leftarrow$ buf $\otimes K$

5. markers $\leftarrow$ NonMaxSuppression $($ buf $)$

Fig. 4. Markers searching algorithm: Symbol $\otimes$ represents convolution operator, symbol \ represents voxel by voxel subtraction of two images. Function called "NonMaxSuppression(buf)" leaves only the local peaks in the image buffer (see [12]). By means of this many redundant markers are suppressed.

The procedure stems from the idea of Hough transform [16] and is similar to the approach used in 5. The main advantage of this approach is its noise robustness. It is well-known that the Hough based methods are very time consuming. Therefore the Fast Fourier Transform [16] was used when implementing the convolution to speed up the whole process. Purposely, we avoid of using mathematical morphology methods 15, such as watershed algorithm, because of the undesirable over-segmentation phenomenon.

Finally the set of markers in space is obtained as a result. If there are some markers too close to each other (closer than a value of $d_{i n}$ ), then the marker with higher quality is left, the other one is removed. 
Deformation. The set of markers and the two parameters $\left(d_{\text {in }}\right.$ and $\left.d_{\text {out }}\right)$ are used to construct the appropriate set of dual simplex meshes. Each dual simplex mesh has its own center and corresponds to one of the markers. All dual simplex meshes are constructed based on the same parameters $-d_{\text {in }}$ and $d_{\text {out }}$. The first one serves for the construction of inner mesh, the second one for the outer mesh. When all the meshes are constructed and consequently composed with image buffers (see Figure 1), the underlying data can be read and the external energies for particular voxels can be precomputed. Now everything is prepared for the deformation.

Deformation is an iterative process. In each step all the objects in space $\mathbb{R}^{3}$ and therefore all the connection lines are exposed to the law of motion. One iteration is equivalent to shortening each of the connection lines. All the connection lines have finite length and the magnitude of the force $\mathbf{F}$ is equivalent to at least one voxel, therefore the iterative process is finite. The deformation continues until some shortening is no longer possible.

Note 3. At this moment the deformation of mesh $D$ is stopped and inner mesh $M$ and outer mesh $M^{\prime}$ are identical. As mentioned above, the dual simplex mesh is tailored for approximation of the spherical-like objects. In general any objects topologically equivalent to a sphere may be approximated with this model. For simplicity of the implementation of initial dual simplex mesh the reconstruction of spherical-like objects was supposed only.

The Evaluation of the Results Quality. The only input parameters, besides image data, given to the algorithm are inner diameter, outer diameter, smoothness parameter and damping factor. It is not obvious to say that the results of the previous step are correct. It's essential to assess the quality of the results.

The assessment is made by measuring some properties of resulting objects (volume, surface, roundness, shape, ....). According to these measures the results are evaluated by the user to be suitable or improper for further processing or another application.

\section{Results and Discussion}

All presented images were cell nuclei whose interior was stained with DAPI. The images were acquired using a confocal microscope Zeiss Axiovert 100 equipped with a CARV confocal unit and a MicroMax CCD camera. The resolution was $0.124 \mu \mathrm{m}$ in the lateral $(x, y)$ direction and $0.3-0.5 \mu \mathrm{m}$ in the axial $(z)$ direction. The images had in average $40 z$-slices and were acquired and stored using FISH 2.0 software package 49. The dimension of processed data was $1300 \times 1030 \times 40$ of voxels. All the computations were performed on Intel Pentium IV $1.4 \mathrm{GHz}$ machine with RAM $256 \mathrm{MB}$ and Red Hat Linux OS.

During the first step of the computation the markers were searched for. (This is the very important part of the algorithm.) If some markers are localized incorrectly or omitted, the consecutive deformation is not able to mend it. During 

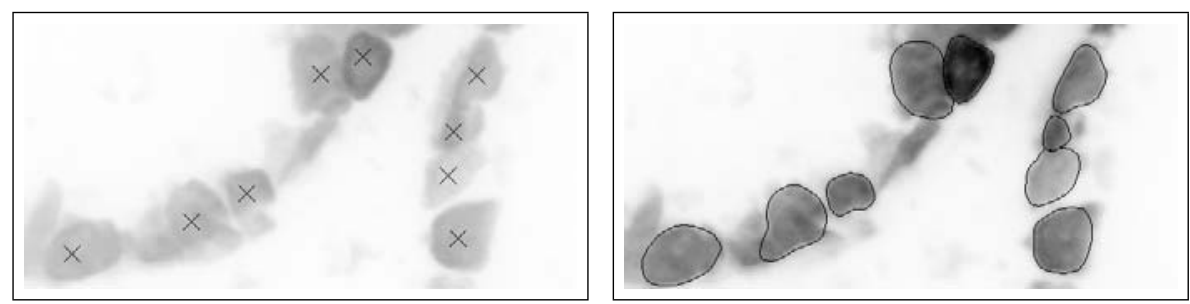

Fig. 5. (left) The input image with overlaid markers, that were found in the first step of the algorithm. Markers are the approximate centers of gravity of studied objects. (right) The results can be stored in many different ways. Drawing contour lines into each slice of source image is one of them. (The depicted contours were obtained by setting the smoothness parameter as well as the damping factor to the value of 0.5 ).

the assessment the objects falsely denoted as cell may be deleted, but those, that are missing, can't be recovered. Many runs of the first part of the algorithm were performed. It was found that the correct setting of the parameters of inner diameter and outer diameter $\left(d_{i n}\right.$ and $d_{\text {out }}$, respectively) is very important. Unfortunately this procedure is highly demanding on the CPU time and memory consuming. More than 1GB of main memory was required at one time. Hence the source images had to be cut into large amount of smaller partially overlapping subimages. Then these were processed consequently without any memory problems. For image data of $1300 \times 1030 \times 40$ dimensions it took 45 mins.

On the other hand a significant acceleration of this process will be possible after distributing the task among two or more computers. Another feature of this step, that is very important, is the noise robustness. When studying procedure in Figure 4, it can be seen that only the gradient image evaluation is noise sensitive. If e. g. Canny edge detector or ISEF (see [14]) is used, the noise is suppressed. In addition the input image was preprocessed (smoothing or median filter) before any computation.

The procedure was tested on different sets of input data. One possible result is depicted in Figure [5 (left). This and many other analogous results were considered by biologists as sufficiently valuable and suitable for next usage.

The deformation step is also noise insensitive [3]. Moreover, it gives very accurate results. It could easily be adjusted by the two parameters: smoothness and damping factor ( $\alpha$ and $\gamma$, respectively). Setting the appropriate values for both of these parameters was simplified by the fact that all the computed energies and consequently the results were normalized. The deformation is stable and it takes only little time to perform the whole computation. The initialization of one cell $\left(d_{\text {out }}<100\right)$ took approximately $230 \mathrm{~ms}$ and the deformation of the same cell took $2.5 \mathrm{sec}$ in average. As could be seen in Figure 5 (right), the results were very accurate and therefore feasible for further computation and measurement.

Theoretically, reconstruction of objects of any size and shape topologically equal to a sphere is possible with using our method. With the apriori knowledge of the likely shape of the reconstructed objects and because of the programming 


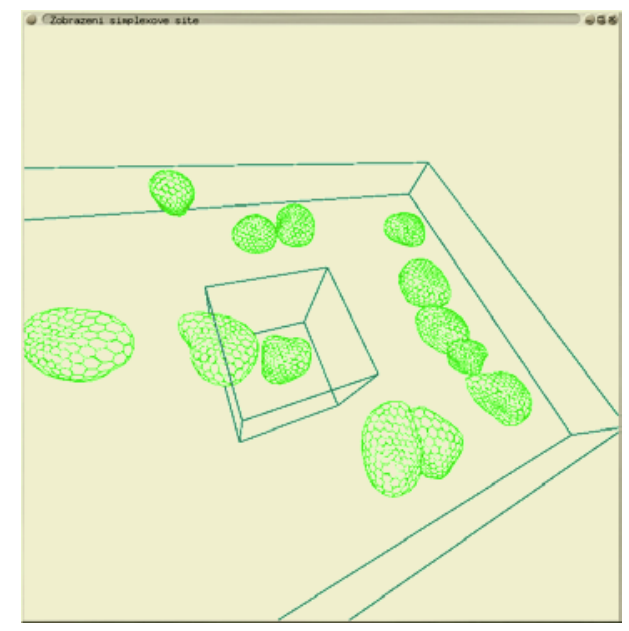

Fig. 6. Reconstructed image visualized using OpenGL library. The large box encloses the image data space. The small box designates one cell in sequence for which the measurement and the evaluation is performed.

simplicity only the sphere-like shaped objects such as cells were submitted to the reconstruction process.

The evaluation step of the algorithm is based on processing the basic measures used in biomedical statistics: volume, surface, roundness, etc. A simple application was implemented for the visualization of the results (see Figure 6). The preliminary results demonstrate that the algorithm converges to the nuclear surface, and that it admits a range of variation in the quality of the staining within and between images. In the forthcoming research some more sophisticated and unsupervised methods for results quality evaluation, such as those published in [11], will be used.

\section{Acknowledgements}

Many thanks are due to I. Koutná who provided large amount of data suitable for testing. This work was supported by the Ministry of Education of the Czech Republic (Project No. MSM-143300002) and by the Academy of Sciences of the Czech Republic (Grants No. S5004010 and No. B5004102).

\section{References}

1. P. Bamford and B. Lovell. Unsupervised Cell Nucleus Segmentation with Active Contours. Signal Processing Special Issue: Deformable Models and Techniques for Image and Signal Processing, vol. 71, p. 203-213, December, 1998

2. H. Delingette. General object reconstruction based on simplex meshes. International Journal of Computer Vision, 32(2):111-146, 1999 
3. P. Matula, D. Svoboda. Spherical Object Reconstruction Using Star-Shaped Simplex Meshes, in Figueiredo M.A.T., Zerubia J., Jain A.K. (Eds.): EMMCVPR 2001, LNCS 2134, pp. 608-620, 2001

4. M. Kozubek, S. Kozubek, E. Lukášová, A. Marečková, E. Bártová, M. Skalníková and A. Jergová. High-resolution cytometry of FISH dots in interphase cell nuclei. Cytometry, vol. 36, p. 279-293, 1999

5. Solorzano C. O., Malladi R., Lelievre S. A. \& Lockett S. J., Segmentation of nuceli and cells using membrane related proteins markers, Journal of Microscopy, Vol. 201, Pt 3, March 2001, pp. 404-415

6. M. Kass, A. Witkin and D. Terzopoulos. Active contour models. International Journal of Computer Vision, 1(4):133-144, 1987

7. H. Netten, I. T. Young, L. J. Van Vliet, H. J. Tanke, H. Vrolijk and W. C. R. Sloos. FISH and chips: automation of fluorescent dot counting in interphase cell nuceli. Cytometry, 28:1-10, 1997

8. J. A. Sethian. Level Set Methods and Fast Marching Methods, Cambridge University Press, 1999

9. M. Kozubek. High-resolution cytometry: Hardware approaches, image analysis techniques and applications, PhD thesis, Masaryk University, Brno, 1998

10. S. R. Gunn and M. S. Nixon. A Dual Active Contour. BMVC 94, September, York, U.K, 305-314, 1994

11. M. Ankerst and G. Kastenmüller and H.-P. Kriegel and T. Seidl. 3D Shape Histograms for Similarity Search and Classification in Spatial Databases, Advances in Spatial Databases, 6th International Symposium, SSD'99, vol. 1651, p. 207-228, 1999

12. F. Devernay. A non-maxima suppression method for edge detection with sub-pixel accuracy. Technical report, INRIA, 1995

13. K. Rodenacker, M. Aubele, P. Hutzler, and U. Adiga P. S. Groping for quantitative digital 3-d image analysis: An approach to quantitative fluorescence in situ hybridization in thick tissue sections of prostate carcinoma. Anal Cell Pathol, 15:19-29, 1997

14. J. R. Parker. Algorithms for Image Processing and Computer Vision, John Wiley \& Sons, NY 1997

15. P. Soille. Morphological image analysis: principles and applications, Springer Verlag, Berlin 1999

16. W. K. Pratt. Digital image processing, 3rd ed., Wiley, New York 2001, ISBN: 0471-37407-5 